\title{
Exact Solutions to the Nonlinear Schrödinger Equation with Time- Dependent Coefficients
}

\author{
Xin-Lei Mai $\mathbb{D}^{1},{ }^{1}$ Wei Li $\mathbb{D},{ }^{1}$ and Shi-Hai Dong $\mathbb{D}^{2,3}$ \\ ${ }^{1}$ School of Mathematics and Physics, Beijing University of Chemical Technology, Beijing 100029, China \\ ${ }^{2}$ Huzhou University, Huzhou 313000, China \\ ${ }^{3}$ CIDETEC, Instituto Politécnico Nacional, Unidad Professional ALM, Mexico City 07700, Mexico
}

Correspondence should be addressed to Xin-Lei Mai; maixinlei7@163.com,Wei Li; liwei2@mail.buct.edu.cn, and Shi-Hai Dong; dongsh2@yahoo.com

Received 9 October 2020; Revised 28 February 2021; Accepted 2 June 2021; Published 16 June 2021

Academic Editor: Osvaldo Civitarese

Copyright (C) 2021 Xin-Lei Mai et al. This is an open access article distributed under the Creative Commons Attribution License, which permits unrestricted use, distribution, and reproduction in any medium, provided the original work is properly cited. The publication of this article was funded by $\mathrm{SCOAP}^{3}$.

\begin{abstract}
In this paper, a trial function method is employed to find exact solutions to the nonlinear Schrödinger equations with high-order time-dependent coefficients. This system might be used to describe the propagation of ultrashort optical pulses in nonlinear optical fibers, with self-steepening and self-frequency shift effects. The new general solutions are found for the general case $a_{0} \neq 0$ including the Jacobi elliptic function solutions, solitary wave solutions, and rational function solutions which are presented in comparison with the previous ones obtained by Triki and Wazwaz, who only studied the special case $a_{0}=0$.
\end{abstract}

\section{Introduction}

It is well known that many physical phenomena can be described by a nonlinear Schrödinger equation (NLSE), which is found in many diverse fields such as plasma physics [1], fluid dynamics [2], nonlinear optics [3], quantum mechanics [4], hydrodynamics [5], and biology. Thus, finding the exact solutions to the NLSE has an important theoretical and practical significance in understanding the physical phenomena described by the NLSE.

Recently, some useful and powerful methods have been proposed to explore its exact solutions. For example, these methods include the homogeneous balance method [6], the $\tan h$ function expansion method and its extension [7], the sine-cosine methods [7], the exp-function method [7], the multiple exp-function method [8], the first integral method [9], the Jacobi elliptic function expansion method [10], the sub-ODE method [11], the $\left(G^{\prime} / G\right)$-expansion method [12], the modified simple equation method [13], the extended auxiliary equation method [14], the $\exp (-\phi(\xi))$-expansion method [15], and the trial function method and its generalization [16].
It should be recognized that most of the methods mentioned above are related to constant coefficient models. Undoubtedly, it becomes more difficult than those constant coefficient counterparts when we study the NLSE with time-dependent coefficients. Up to now, considerable attention has been paid to the varying coefficient NLSE, and many authors put forward different approaches [16-22]. Among them, Liu [21] proposed a trial function method to deal with both real and complex equations with varying coefficients. In this paper, we are going to apply Liu's method to find the exact solutions of the following cubic-quintic NLSE with time-dependent coefficients:

$i q_{t}+f(t) q_{x x}+g(t)\left(|q|^{2}+\sigma|q|^{4}\right) q=i h(t)\left(|q|^{2} q\right)_{x}+i p(t)\left(|q|^{2}\right)_{x} q$.

$q(x, t)$ will represent a different physical quantity when using it to describe different systems. For example, when the present equation with high-order dispersion and nonlinear terms describes the pulse transmission in the femtosecond state and considers the loss in the transmission process 


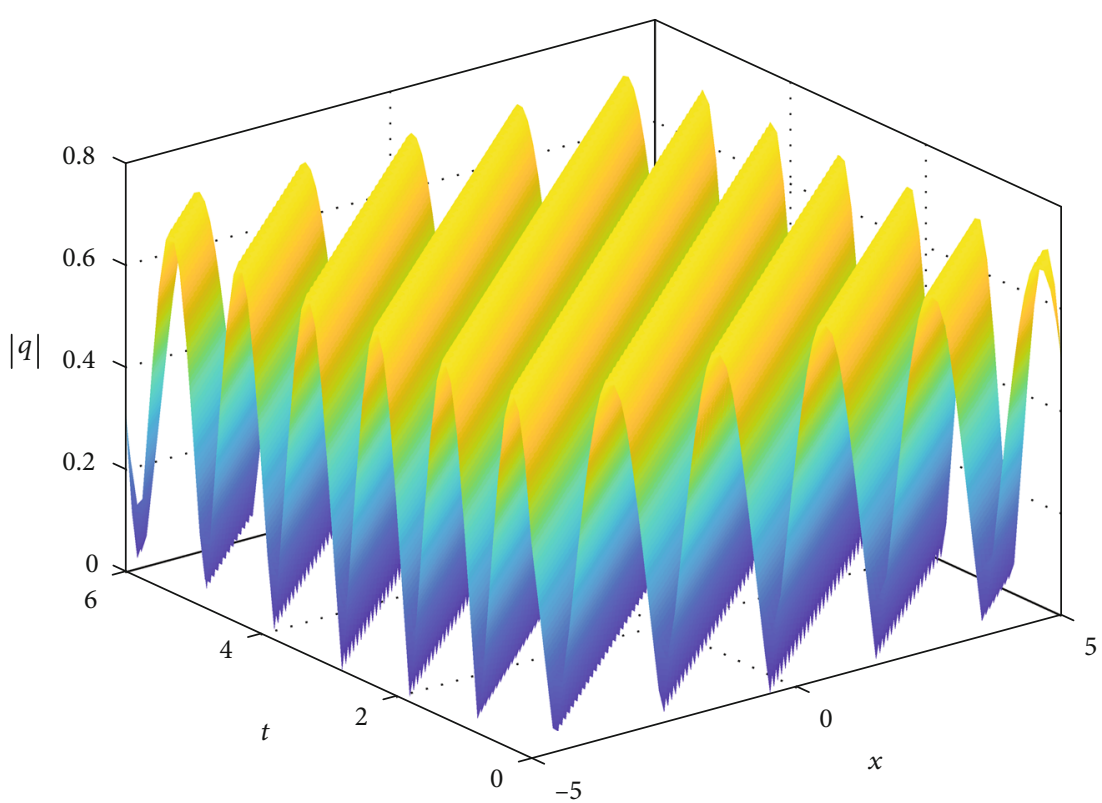

(a)

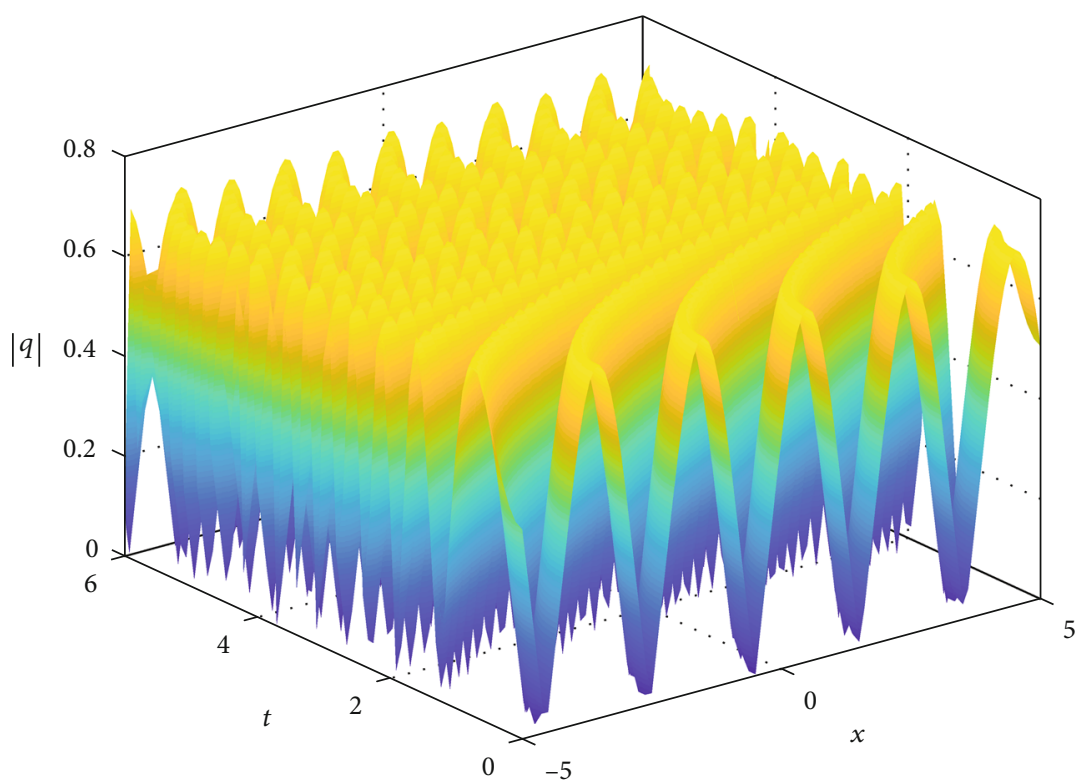

(b)

Figure 1: Continued. 


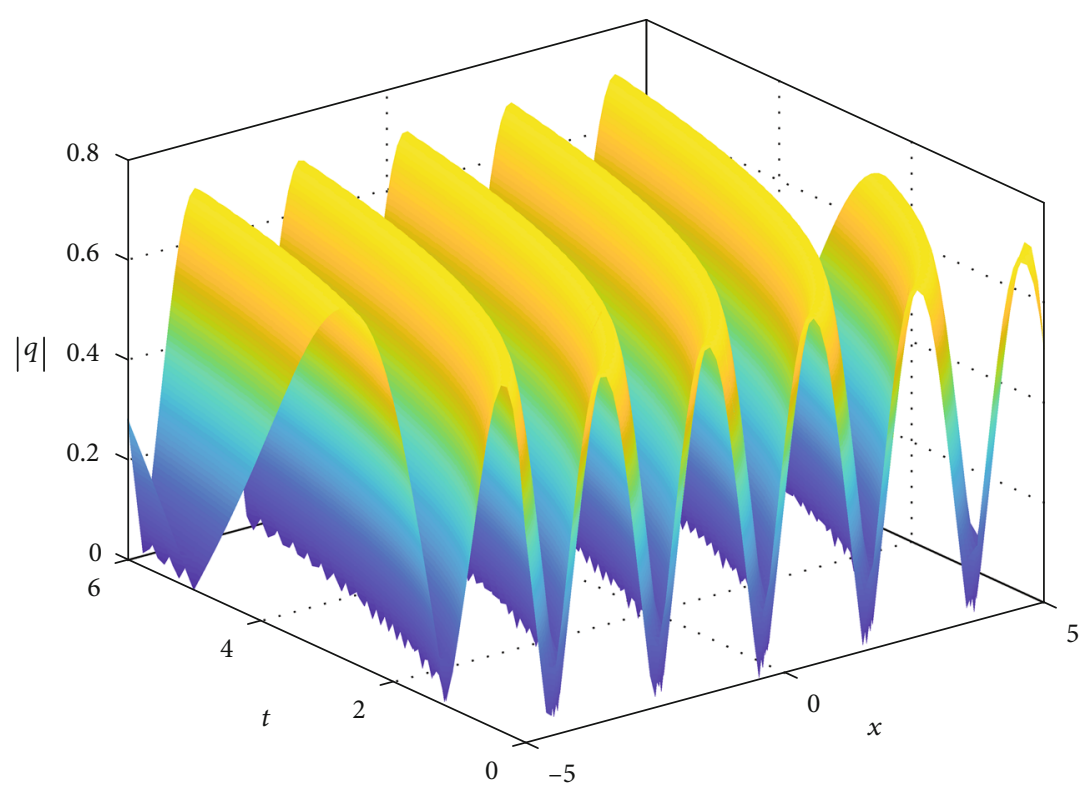

(c)

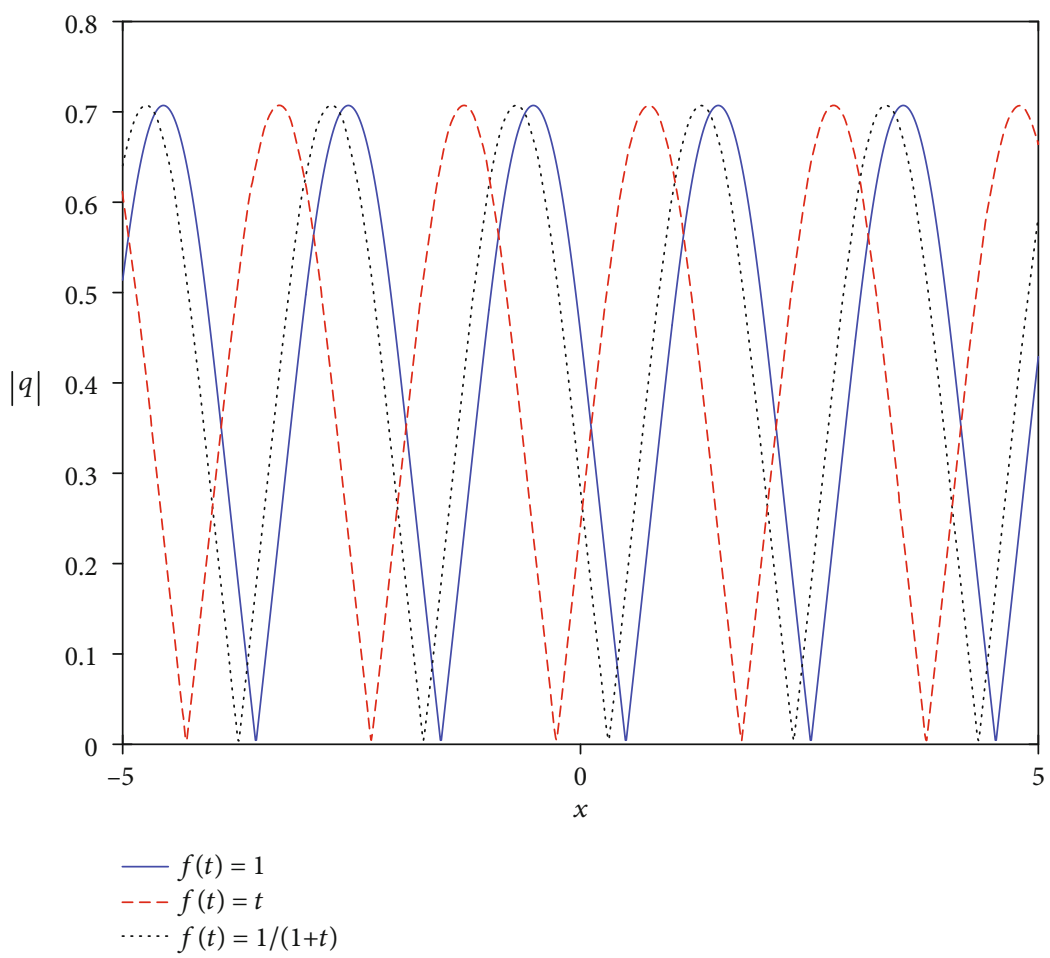

(d)

Figure 1: (a-c) The 3D wave shapes of $|q|:$ (a) $f(t)=1$, (b) $f(t)=t$, and (c) $f(t)=1 /(1+t)$. (d) The wave propagates along the $x$-axis.

$[17,22], q(x, t)$ denotes the complex envelope of the electric field, $x$ and $t$ are the distance along the direction of propagation and time, respectively, $f(t)$ is the dispersion coefficient, $h(t)$ is the self-steepening coefficient, $p(t)$ is the selffrequency shift coefficient, and $\sigma$ is a constant. Green and Biswas [22] studied Equation (1) by using the ansatz method [23] and obtained the exact soliton solutions under some constraints on the parameters. Recently, one of the present authors has obtained analytical traveling-wave solutions to a generalized Gross-Pitaevskii (GP) equation [22] with some new time- and space-varying coefficients and external fields [24] because of their possible applications to the BECs [2529]. Obviously, the current cubic-quintic NLSE is more complicated than the GP equation. It should be pointed out that all kinds of NLSEs with varying coefficients mentioned above [16-22] are different from Equation (1) except for reference 


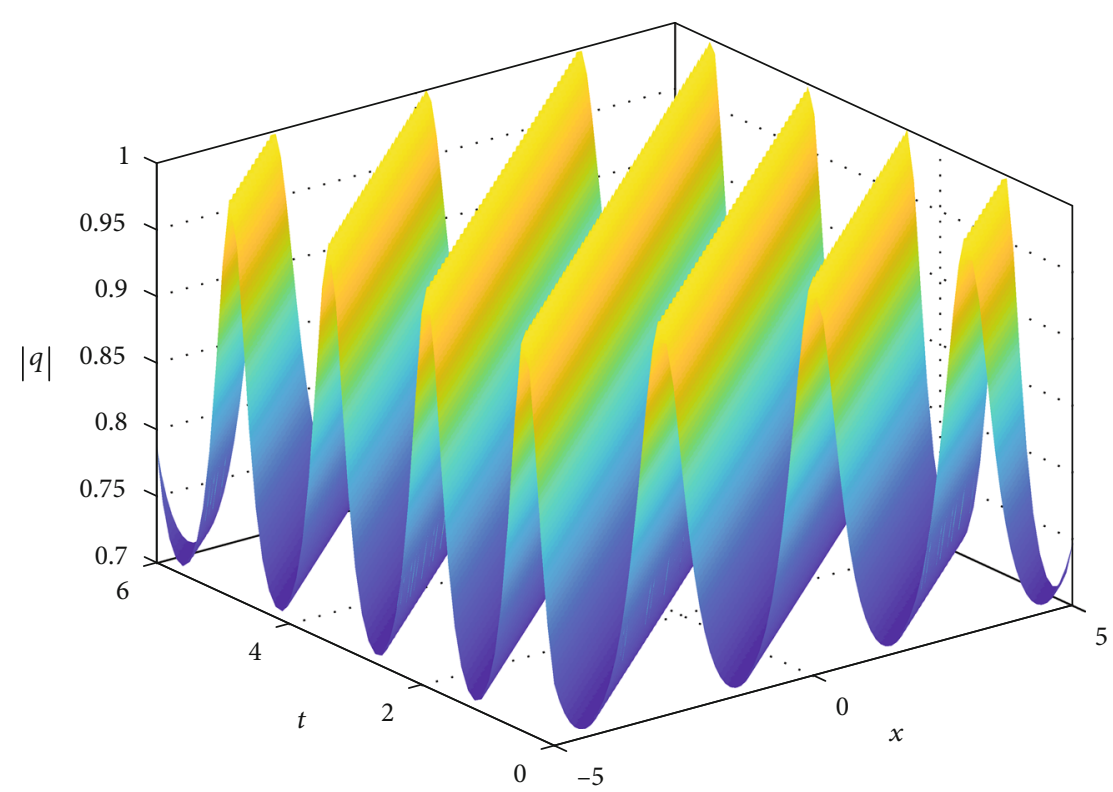

(a)

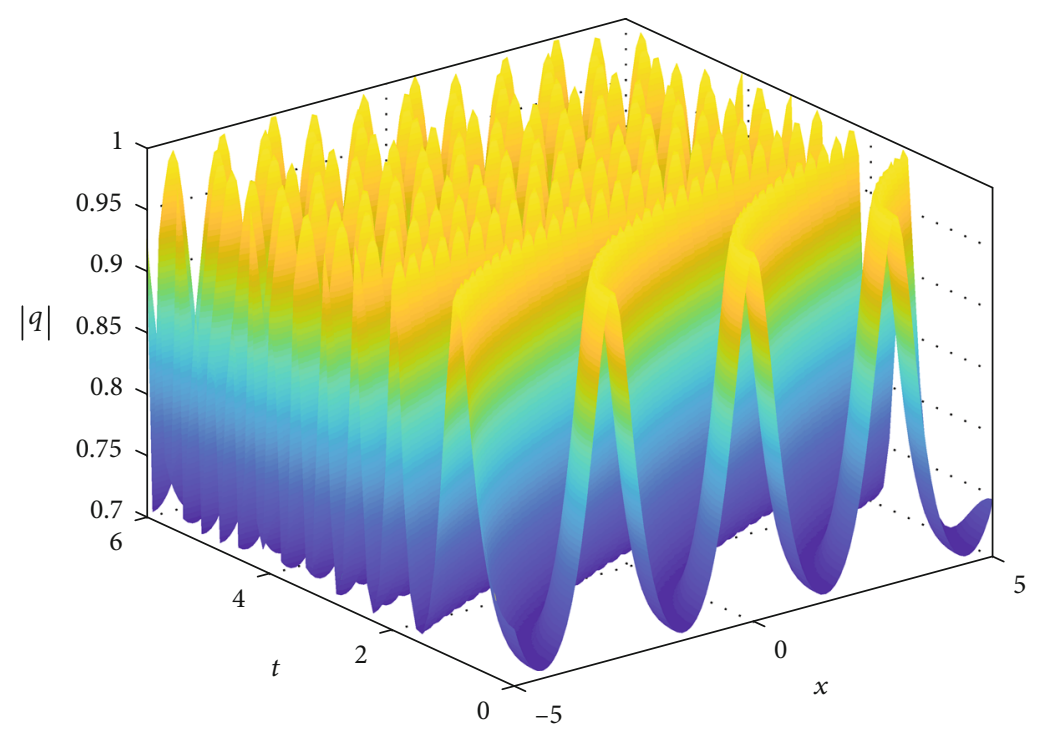

(b)

Figure 2: Continued. 


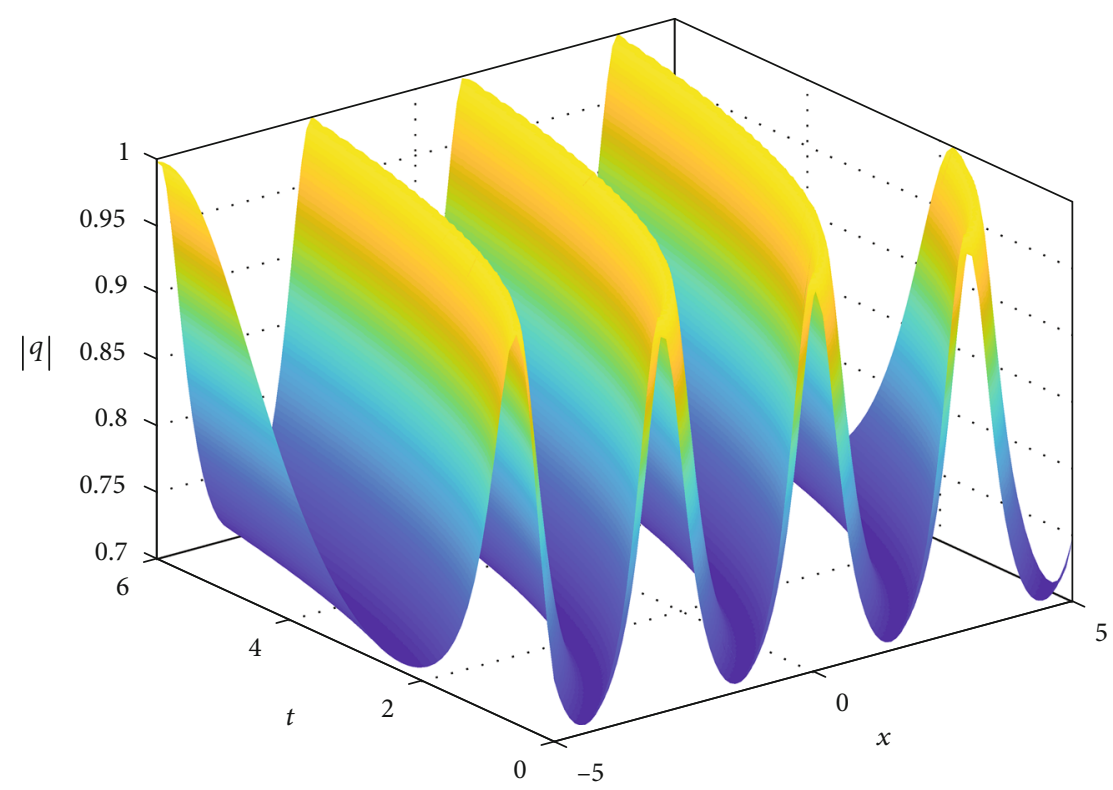

(c)

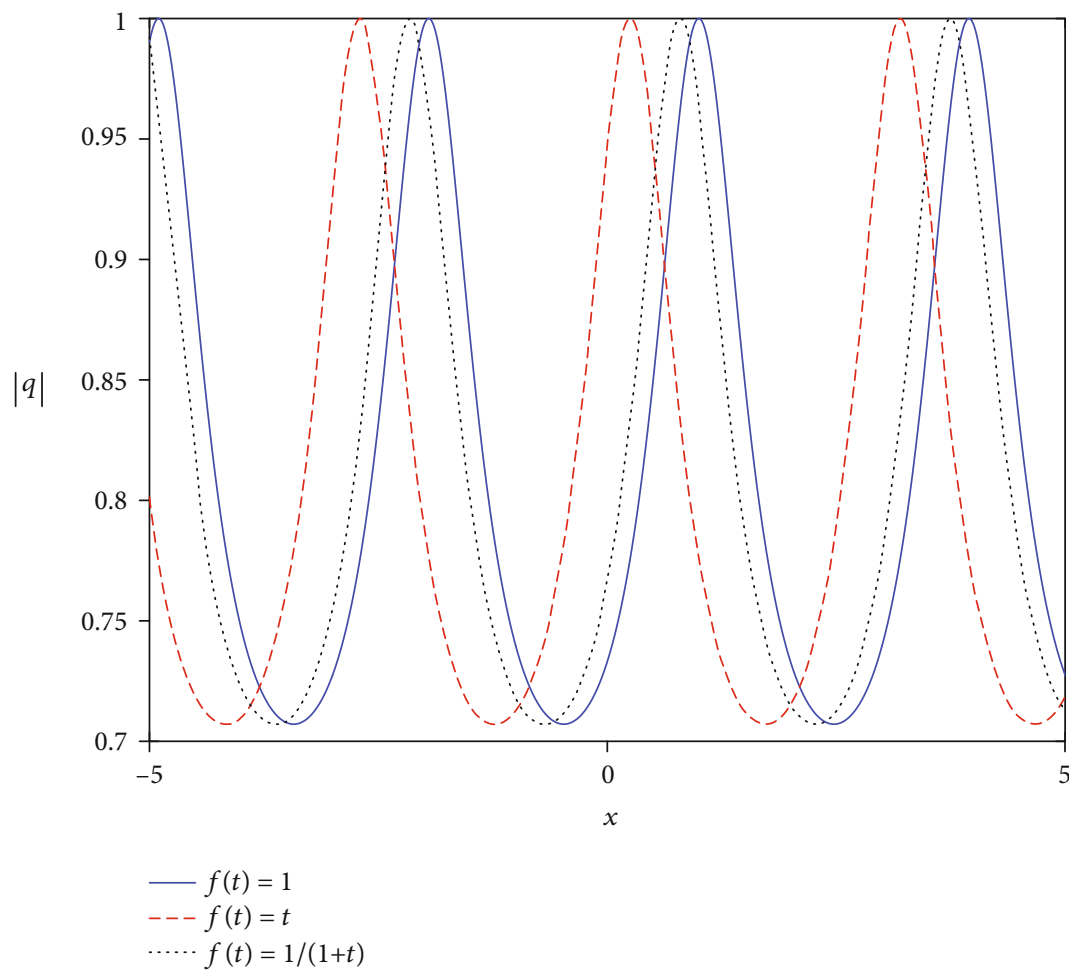

(d)

FIgURe 2: $(\mathrm{a}-\mathrm{c})$ The 3D wave shapes of $|q|:(a) \mathrm{f}(\mathrm{t})=1$, (b) $\mathrm{f}(\mathrm{t})=\mathrm{t}$, and $(\mathrm{c}) \mathrm{f}(\mathrm{t})=1 /(1+\mathrm{t})$. (d) The wave propagates along the $x$-axis.

[17], in which the authors only studied the particular case $a_{0}=0$ using the direct but complicated integral approach.

This paper is organized as follows. In "Exact Solutions," we first apply the trial function method to obtain its exact solutions by using a suitable transformation, and then, we illustrate the shapes of the wave amplitude of different solutions by taking appropriate parameters for those varying coefficients. Finally, in "Concluding Remarks," we summarize the results found in this work.

\section{Exact Solutions}

Assume that the solution to Equation (1) is given by

$$
q(x, t)=u(\xi) e^{i \eta}, \xi=k(t) x+w(t), \eta=s(t) x+r(t)
$$

where $k(t), w(t), s(t)$, and $r(t)$ are undetermined parameters related to time. Substituting them into (1) and separating the real and the imaginary parts, one finds 


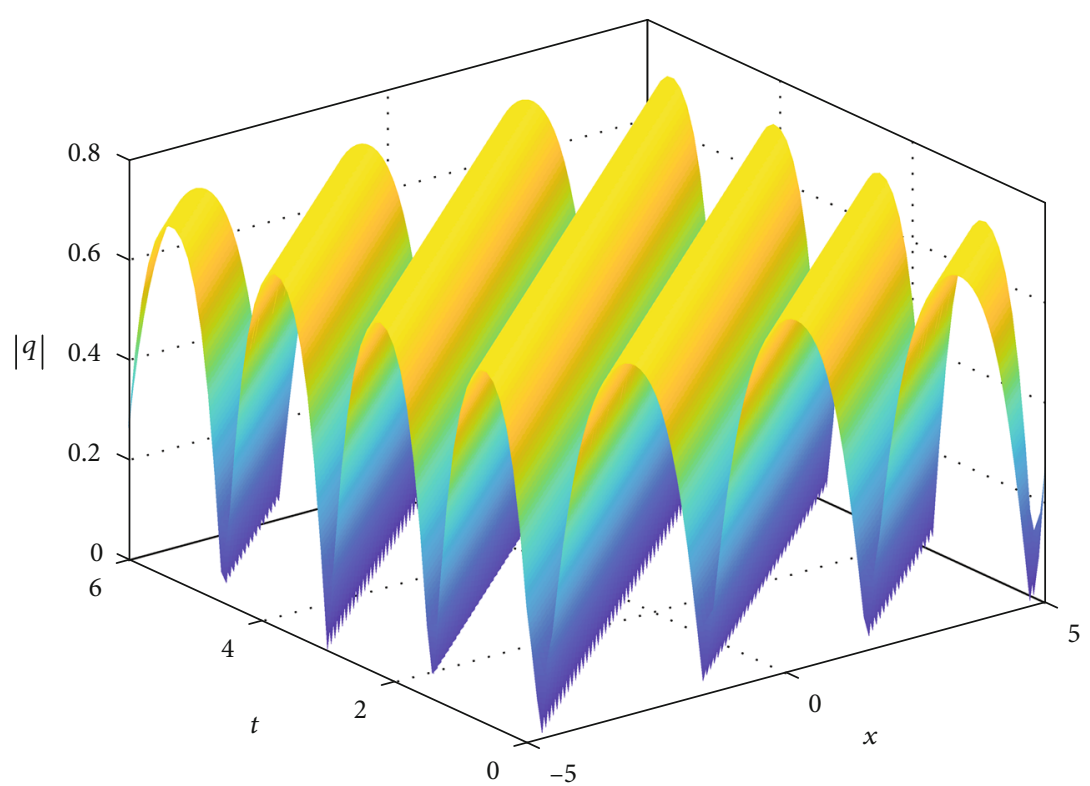

(a)

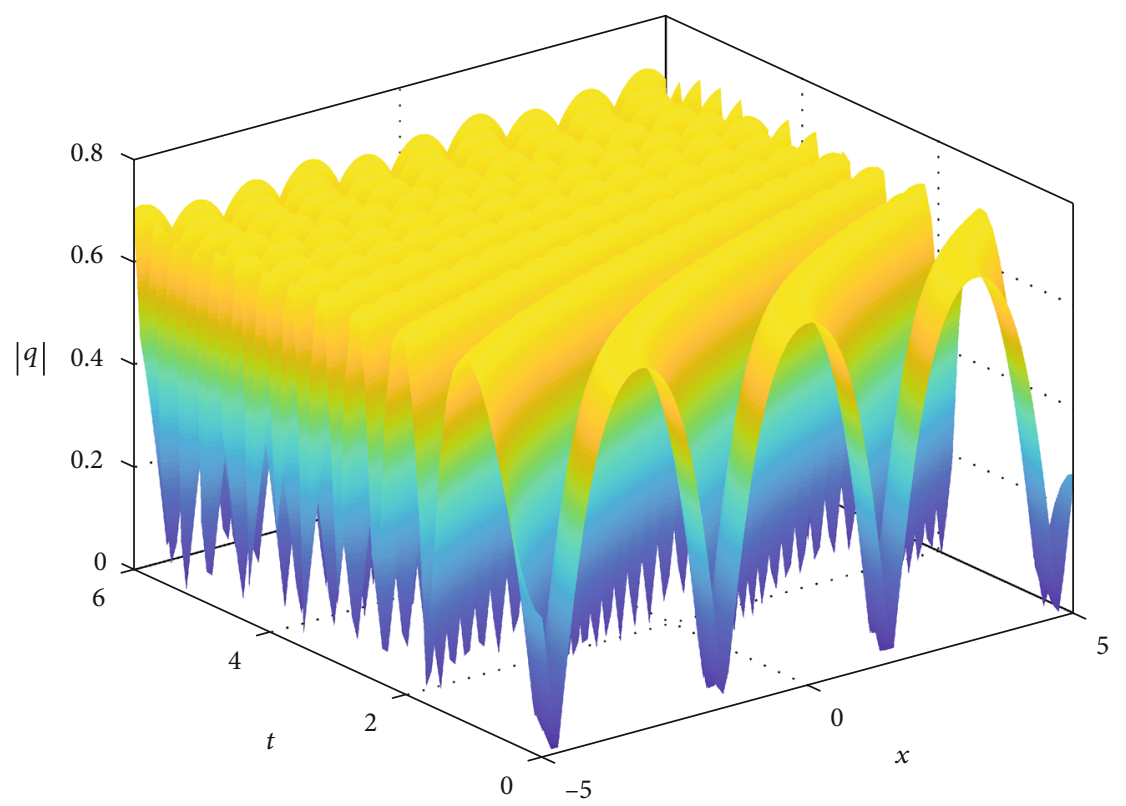

(b)

Figure 3: Continued. 


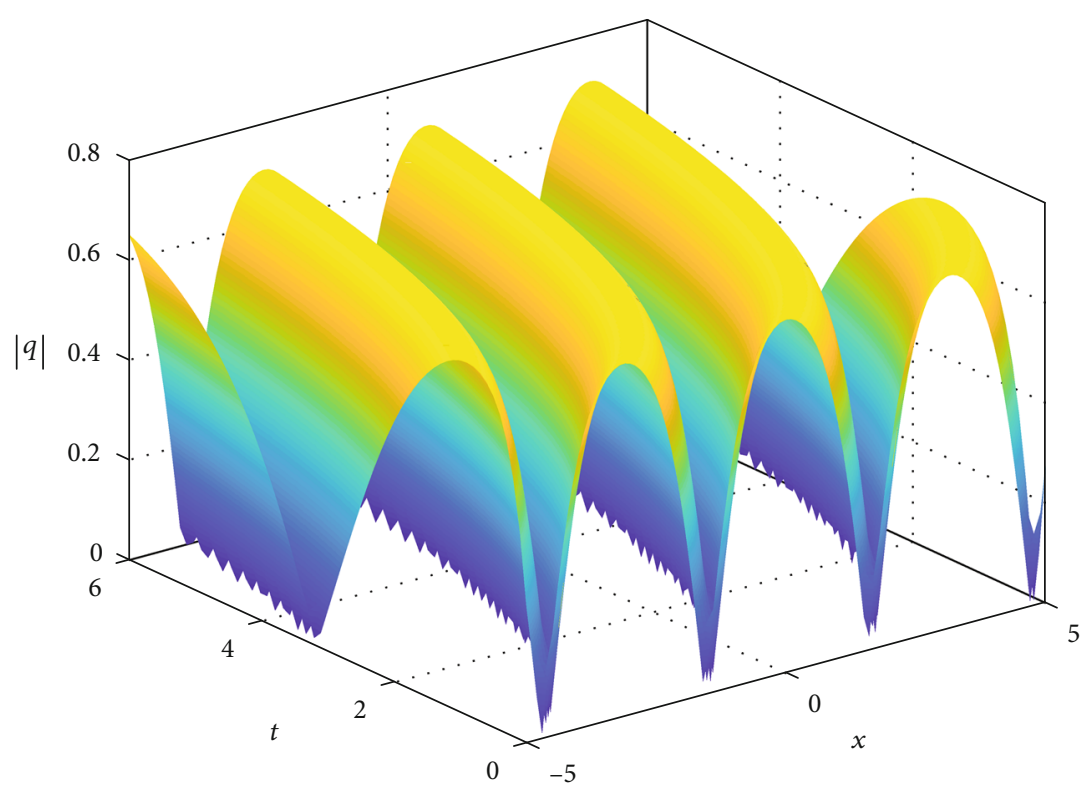

(c)

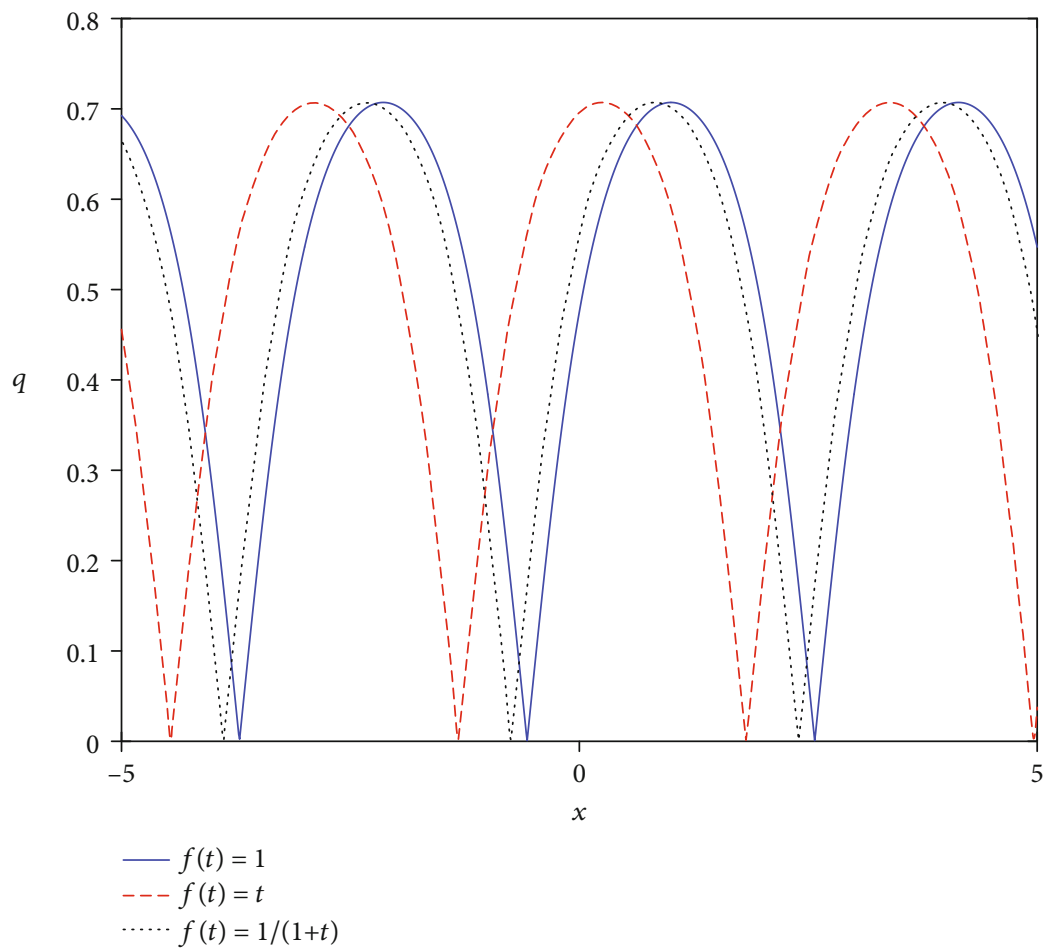

(d)

Figure 3: (a-c) The 3D wave shapes of $|q|$ : (a) $f(t)=1$, (b) $f(t)=t$, and (c) $f(t)=1 /(1+t)$. (d) The wave propagates along the $x$-axis.

$$
\begin{gathered}
{\left[k^{\prime}(t) x+w^{\prime}(t)+2 f(t) s(t) k(t)\right] u^{\prime}-[3 h(t)+2 p(t)] k(t)} \\
f(t) k^{2}(t) u^{\prime \prime}-\left[s^{\prime}(t) x+r^{\prime}(t)+f(t) s^{2}(t)\right] u \\
+[g(t)+h(t) s(t)] u^{3}+\sigma g(t) u^{5}=0 .
\end{gathered}
$$

If the solution satisfies

$$
\left(u^{\prime}\right)^{2}=F(\xi)=\sum_{i=0}^{m} a_{i} u^{i},
$$

where $a_{i}(i=0, \cdots, m)$ are constants and $m$ is an integer to be determined (the value of $m$ in Eq. (5) is determined as $m=6$ when using the homogeneous balance theory), then substituting (5) into (4) and setting each coefficient of $u^{\prime}$, 
$u^{2} u^{\prime}$ in (3), and $u^{i}(i=1,3,5)$ in (4) to zero allow us to obtain a set of algebraic equations as follows:

$$
\begin{gathered}
k^{\prime}(t) x+w^{\prime}(t)+2 f(t) s(t) k(t)=0, \\
{[3 h(t)+2 p(t)] k(t)=0, \quad a_{1}=a_{3}=a_{5}=0,} \\
a_{2}=\frac{s^{\prime}(t) x+r^{\prime}(t)+f(t) s^{2}(t)}{f(t) k^{2}(t)}, \\
a_{4}=-\frac{g(t)+h(t) s(t)}{2 f(t) k^{2}(t)} \\
a_{6}=-\frac{\sigma g(t)}{3 f(t) k^{2}(t)}
\end{gathered}
$$

Set

$$
\begin{aligned}
k(t) & =k \neq 0, s(t)=s \neq 0, r(t)=c_{1} \int f(t) d t, g(t) \\
& =c_{2} k^{2} f(t), h(t)=c_{3} k^{2} f(t), a_{0}=c_{4},
\end{aligned}
$$

where $k, s, c_{1}, c_{2}, c_{3}$, and $c_{4}$ are arbitrary constants, $f(t)$ is an arbitrary function. In this work, we only consider three different cases, $f(t)=1, f(t)=t$, and $f(t)=1 /(1+t)$, for sim- plicity. The other coefficients can be determined by the following forms:

$$
\begin{aligned}
p(t) & =-\frac{3}{2} c_{3} k^{2} f(t), \\
w(t) & =-2 s k \int f(t) d t, \\
a_{1} & =a_{3}=a_{5}=0, \\
a_{2} & =\frac{c_{1}+s^{2}}{k^{2}} \\
a_{4} & =-\frac{c_{2}+s c_{3}}{2}, \\
a_{6} & =-\frac{\sigma c_{2}}{3}
\end{aligned}
$$

In terms of these coefficients (8), Equation (5) with $m=6$ is thus simplified as

$$
\left(u^{\prime}\right)^{2}=a_{0}+a_{2} u^{2}+a_{4} u^{4}+a_{6} u^{6}
$$

Before studying the general case $a_{0} \neq 0$, let us first show the soliton solutions $q=u(\xi) e^{i \eta}$ for the special case $a_{0}=0$ [17]:

$$
q=\left\{\begin{array}{l}
{\left[\frac{2 a_{2}}{\varepsilon \sqrt{a_{4}^{2}-4 a_{2} a_{6}} \cos h\left(2 \sqrt{a_{2}} \xi\right)-a_{4}}\right]^{1 / 2} \cdot e^{i \eta}, \quad a_{4}^{2}-4 a_{2} a_{6}>0, \quad a_{2}>0, \quad \text { bright soliton solution, }} \\
{\left[\frac{2 a_{2}}{\varepsilon \sqrt{4 a_{2} a_{6}-a_{4}^{2}} \sin h\left(2 \sqrt{a_{2}} \xi\right)-a_{4}}\right]^{1 / 2} \cdot e^{i \eta}, \quad a_{4}^{2}-4 a_{2} a_{6}<0, \quad a_{2}>0, \quad \text { singular soliton solution, }} \\
{\left[-\frac{a_{2}}{a_{4}}\left(1+\varepsilon \tan h\left(\frac{\sqrt{a_{2}}}{2} \xi\right)\right)\right]^{1 / 2} \cdot e^{i \eta}, \quad a_{4}^{2}-4 a_{2} a_{6}=0, \quad a_{2}>0, \quad \text { dark soliton solution, }} \\
{\left[\frac{-a_{2} a_{4} \sec h^{2}\left(\sqrt{a_{2}} \xi\right)}{a_{4}^{2}-a_{2} a_{6}\left[1+\varepsilon \tan h\left(\sqrt{a_{2}} \xi\right)\right]^{2}}\right]^{1 / 2} \cdot e^{i \eta}, \quad a_{2}>0, \quad \varepsilon= \pm 1, \quad \text { bright soliton-like solution. }}
\end{array}\right.
$$

How to find the exact solution to the general case $a_{0} \neq 0$ becomes the main purpose of this work. Applying a transformation $\varphi=u^{-2}$ to Equation (10) enables us to obtain

$$
\varphi^{\prime}= \pm 2 \sqrt{a_{0} G(\varphi)}, G(\varphi)=\varphi^{3}+\frac{a_{2}}{a_{0}} \varphi^{2}+\frac{a_{4}}{a_{0}} \varphi+\frac{a_{6}}{a_{0}} .
$$

It is found that Equation (12) can be divided into three different cases depending on the factor $\Delta=B^{2}-4 A C$ by the Shengjin discrimination method, where the parameters $A, B$, and $C$ are given by

$$
\begin{aligned}
& A=\frac{a_{2}^{2}-3 a_{0} a_{4}}{a_{0}^{2}}, \\
& B=\frac{a_{2} a_{4}-9 a_{0} a_{6}}{a_{0}^{2}}, \\
& C=\frac{a_{4}^{2}-3 a_{2} a_{6}}{a_{0}^{2}} .
\end{aligned}
$$

Case 1. $\Delta<0$ 


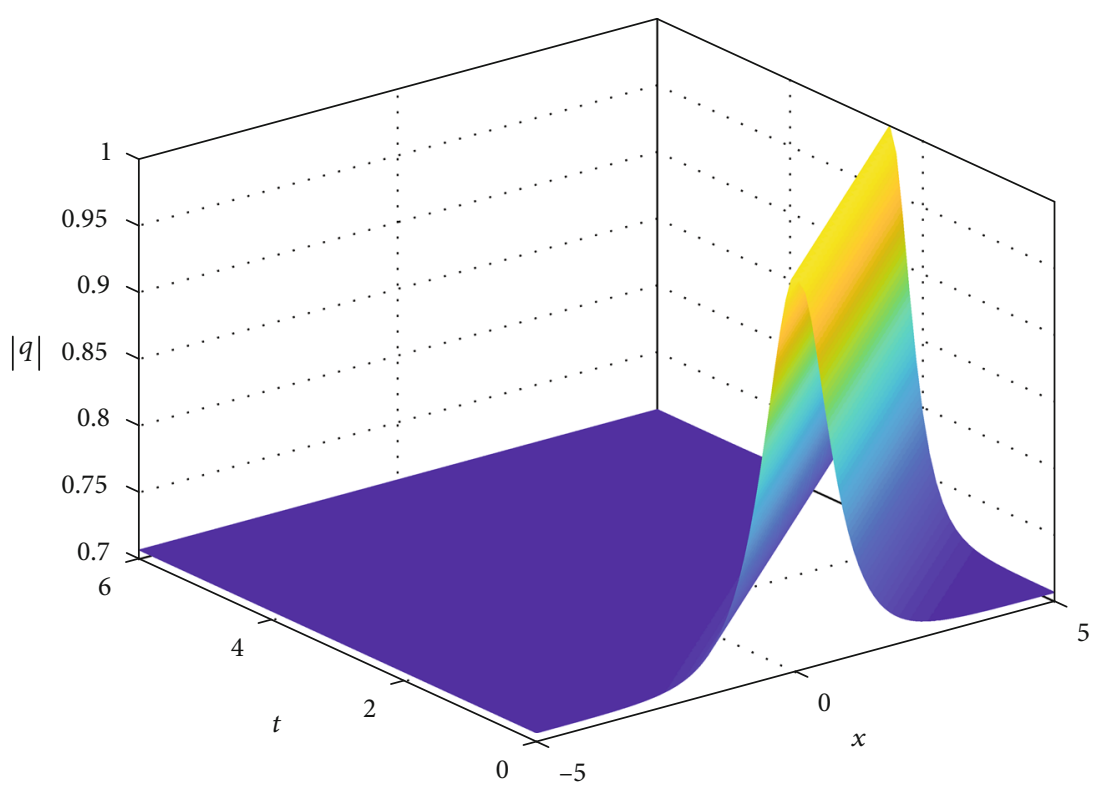

(a)

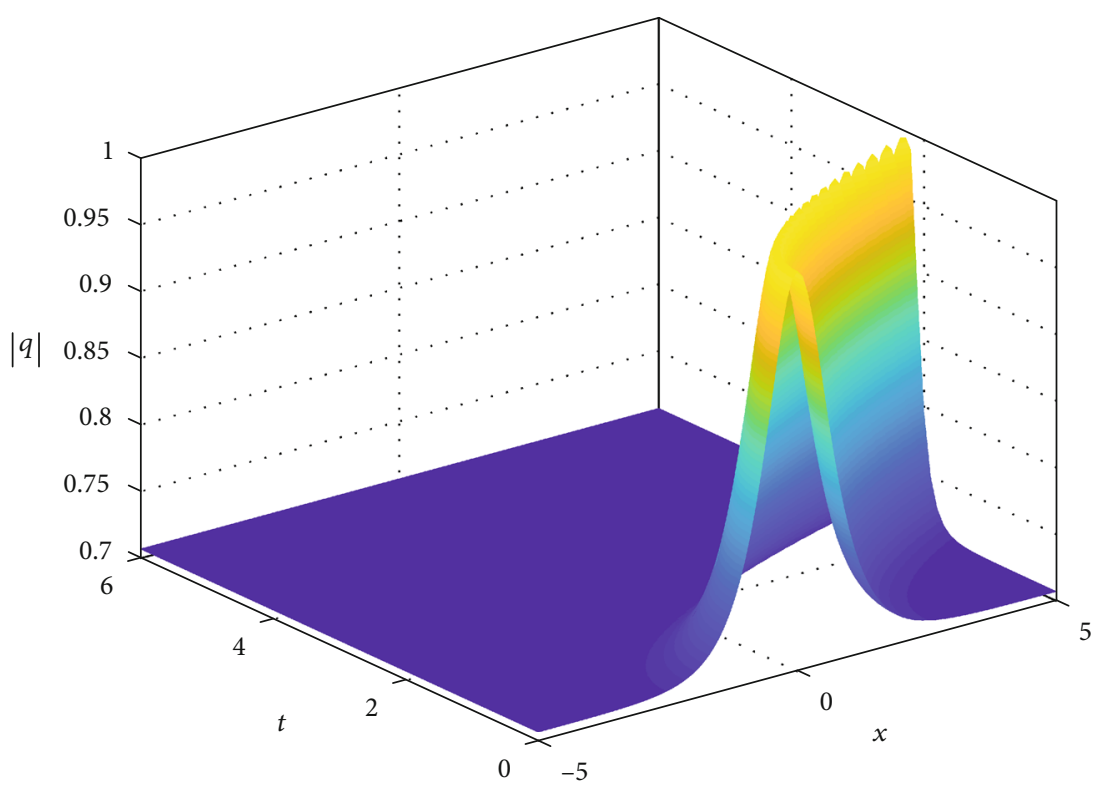

(b)

FIgure 4: Continued. 


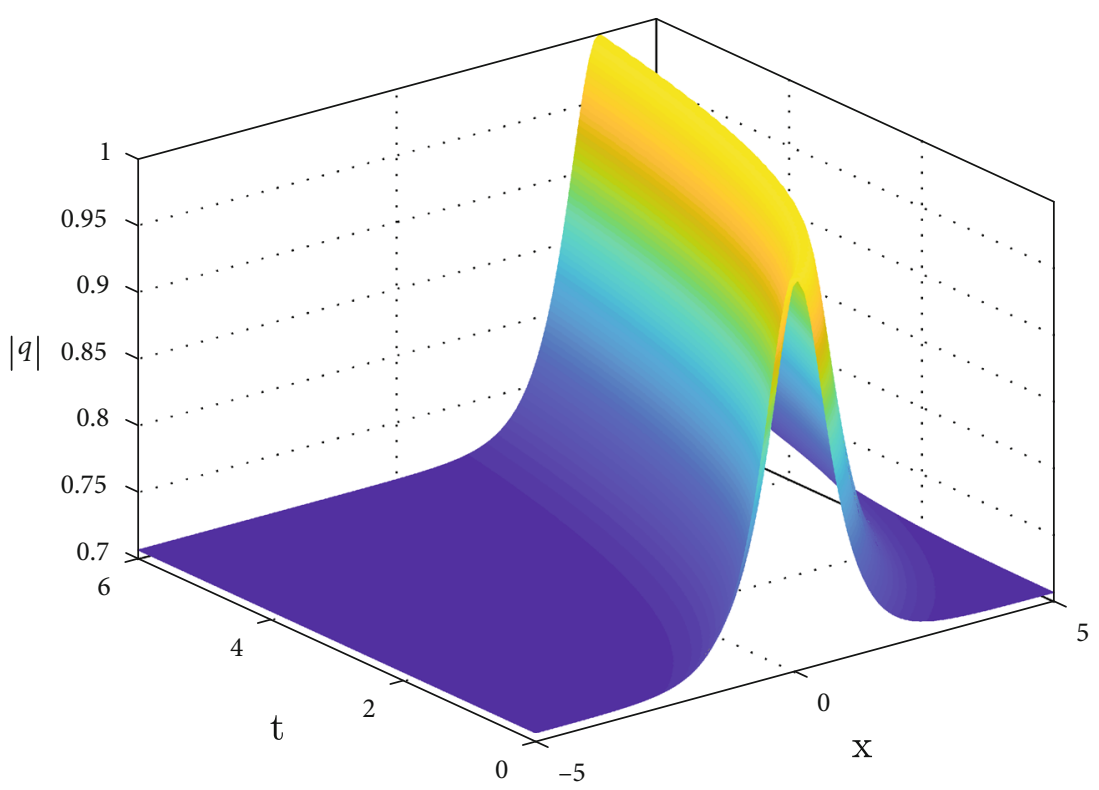

(c)

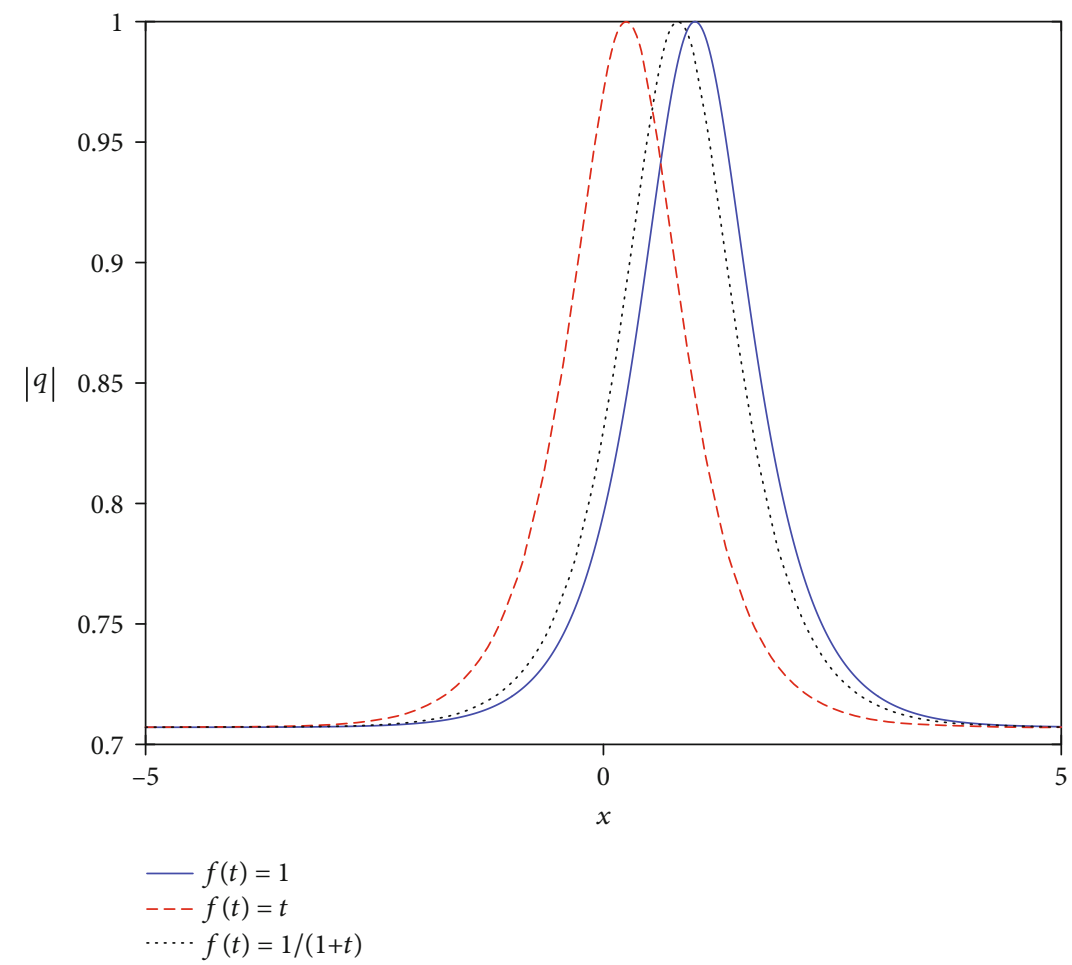

(d)

Figure 4: (a-c) The 3D wave shapes of $|q|$ : (a) $f(t)=1$, (b) $f(t)=t$, and (c) $f(t)=1 /(1+t)$. (d) The wave propagates along the $x$-axis.

In this case, $G(\varphi)=0$ has three unequal roots, $\varphi_{1}<\varphi_{2}<$ $\varphi_{3}: \varphi_{1}=-a_{2} / 3 a_{0}-2 \sqrt{A} \cos (\theta / 3) / 3$ and $\varphi_{2,3}=-a_{2} / 3 a_{0}+$ $\sqrt{A}[\cos (\theta / 3) \pm \sqrt{3} \sin (\theta / 3)] / 3$, where $\theta=\arccos \left(\left(2 A a_{2}-3\right.\right.$ $\left.\left.a_{0} B\right) / 2 a_{0} \sqrt{A^{3}}\right)$. In terms of them, Equation (12) can be expressed as $\varphi^{\prime}= \pm 2 \sqrt{a_{0}\left(\varphi-\varphi_{1}\right)\left(\varphi-\varphi_{2}\right)\left(\varphi-\varphi_{3}\right)}$. According to the relation $\varphi=u^{-2}$, a periodic wave solution $q(x, t)$ $=u(\xi) e^{i \eta}$ of Equation (1) is found as

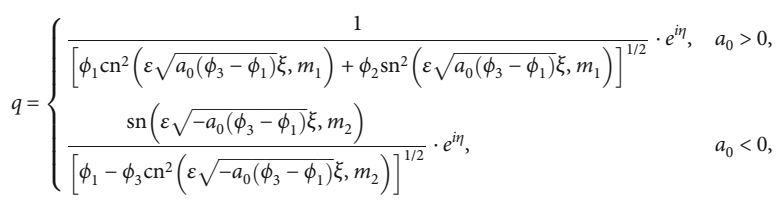

where 


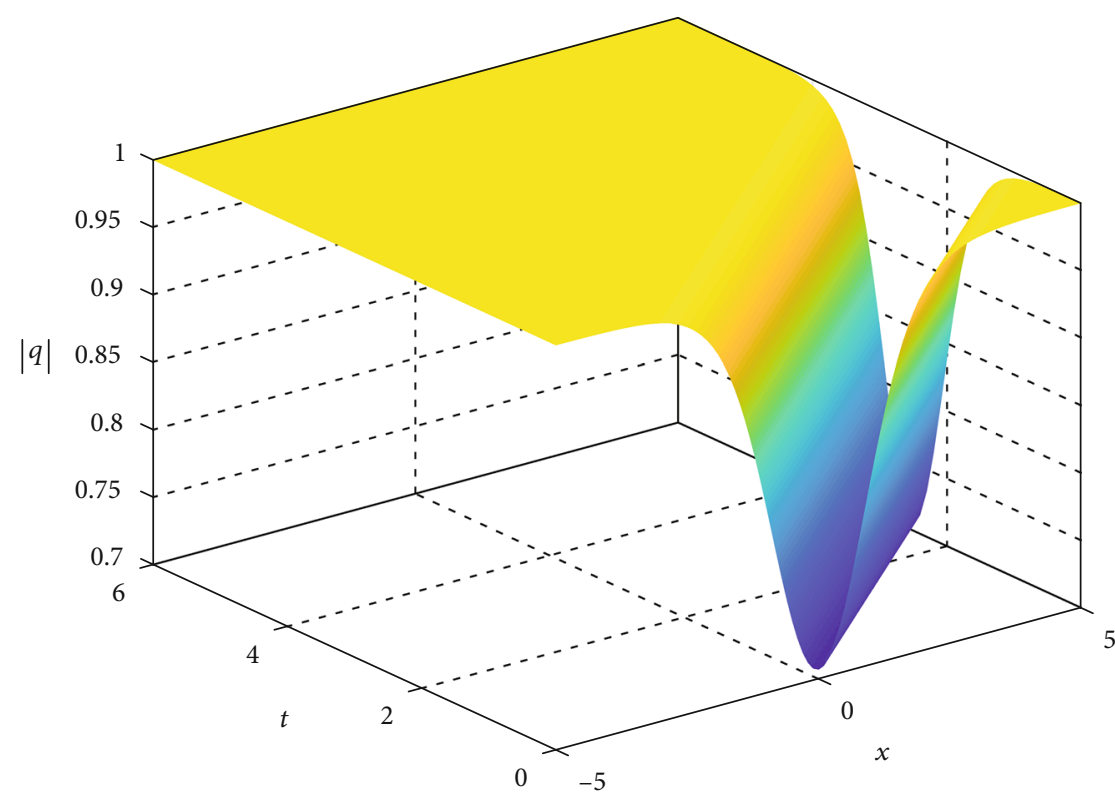

(a)

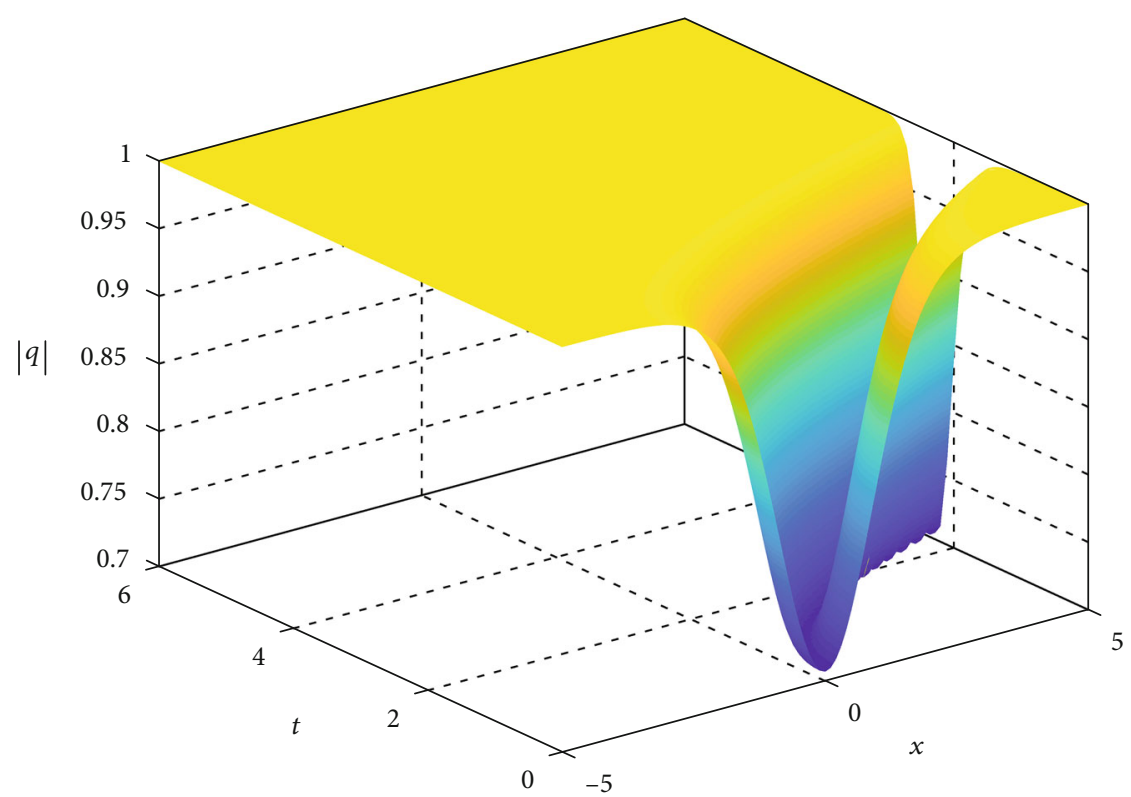

(b)

Figure 5: Continued. 


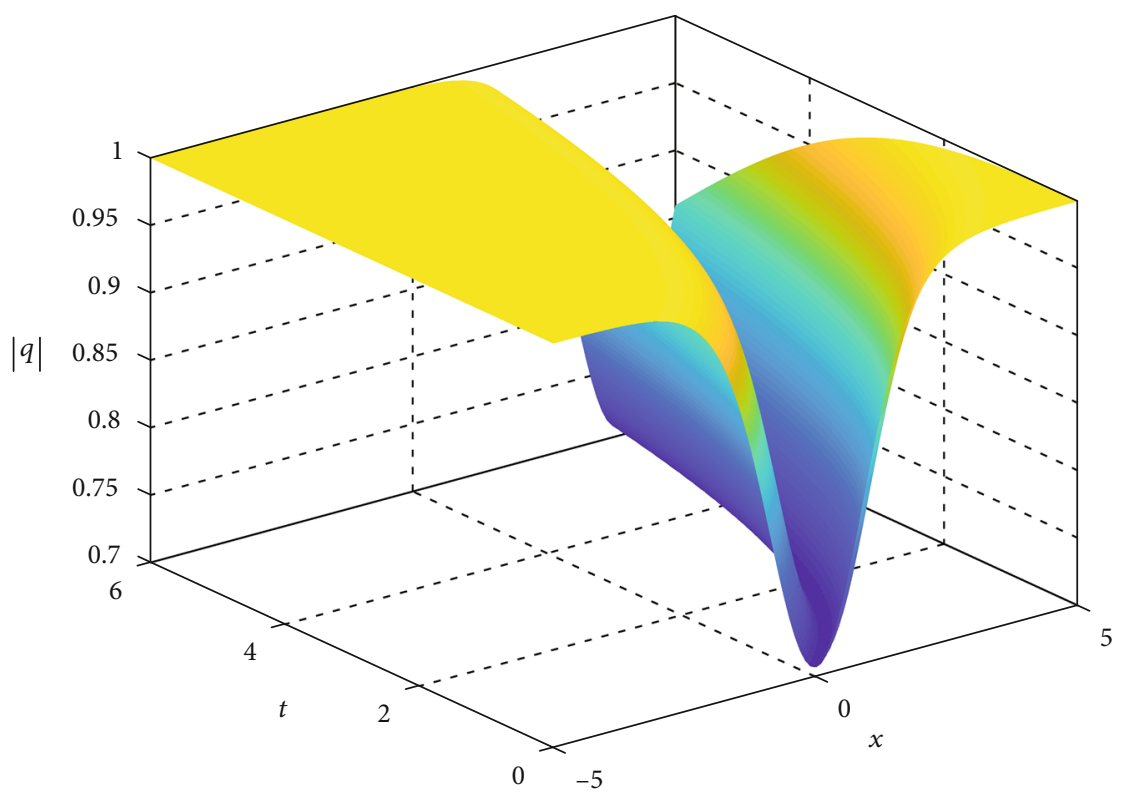

(c)

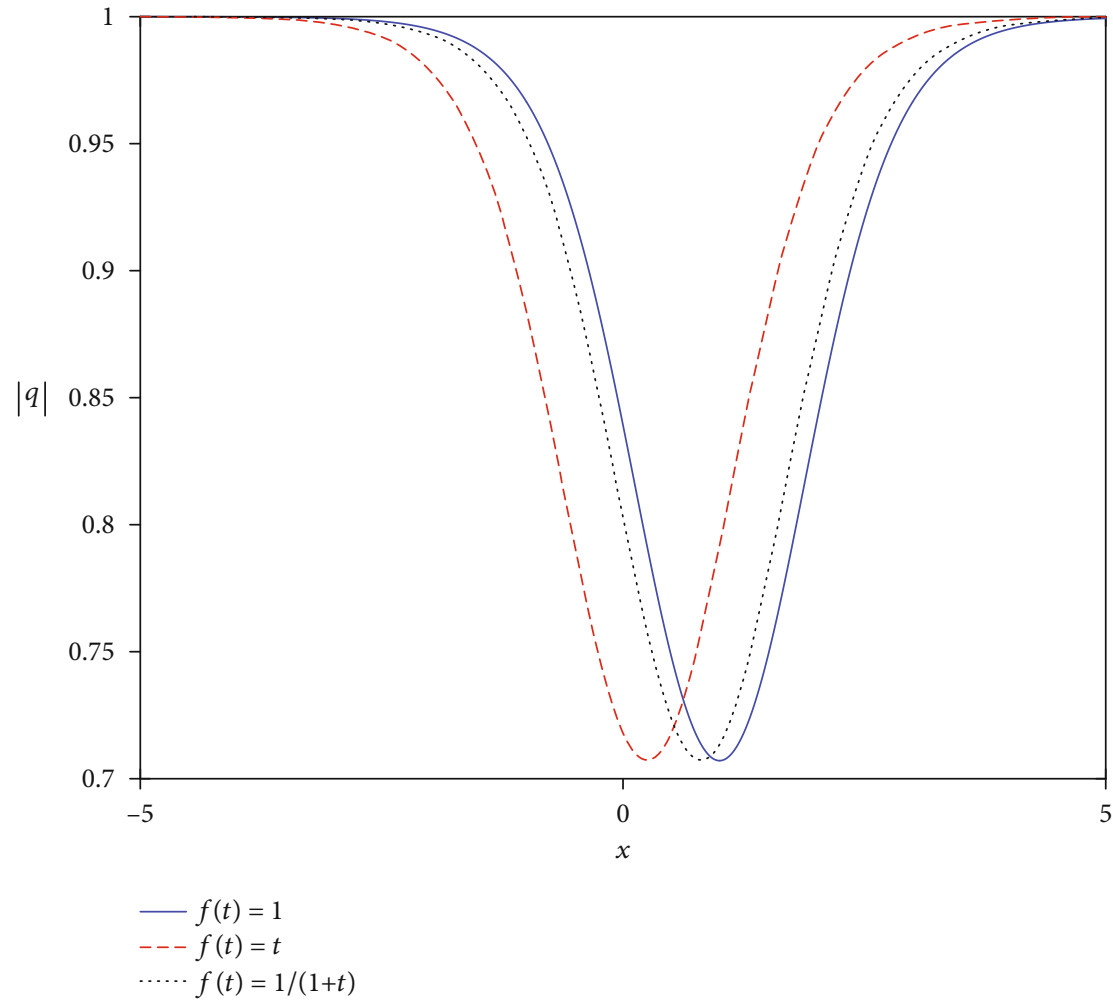

(d)

Figure 5: (a-c) The 3D wave shapes of $|q|:(\mathrm{a}) f(t)=1$, (b) $f(t)=t$, and (c) $f(t)=1 /(1+t)$. (d) The wave propagates along the $x$-axis.

$$
\begin{aligned}
& m_{1}=\sqrt{\frac{\phi_{2}-\phi_{1}}{\phi_{3}-\phi_{1}}}, \\
& m_{2}=\sqrt{\frac{\phi_{3}-\phi_{2}}{\phi_{3}-\phi_{1}}} .
\end{aligned}
$$

To understand these solutions, we illustrate the shapes of the wave amplitude for three different cases, $f(t)=1, f(t)=t$, and $f(t)=1 /(1+t)$, which are all periodic waves $(k=s=1$, $\left.a_{0}=1, a_{2}=-6, a_{4}=11, a_{6}=-6\right)$.

Case 2. $\Delta>0$ 


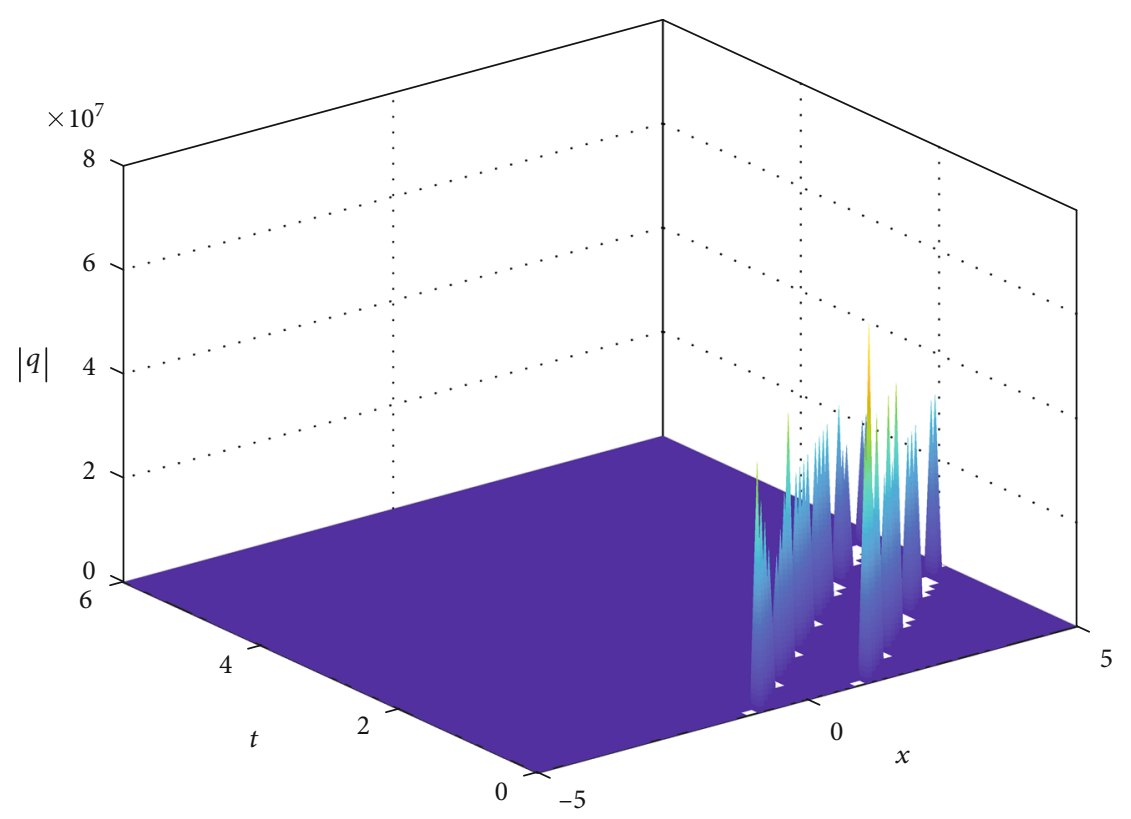

(a)

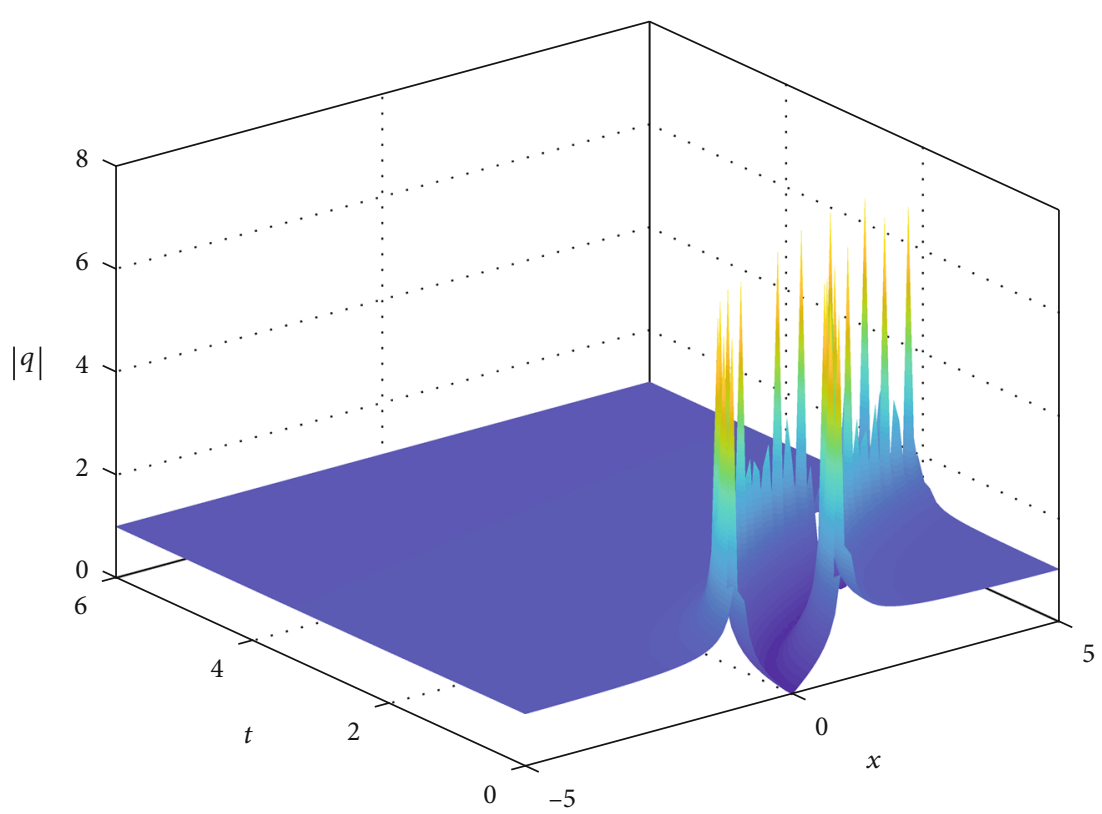

(b)

Figure 6: Continued. 


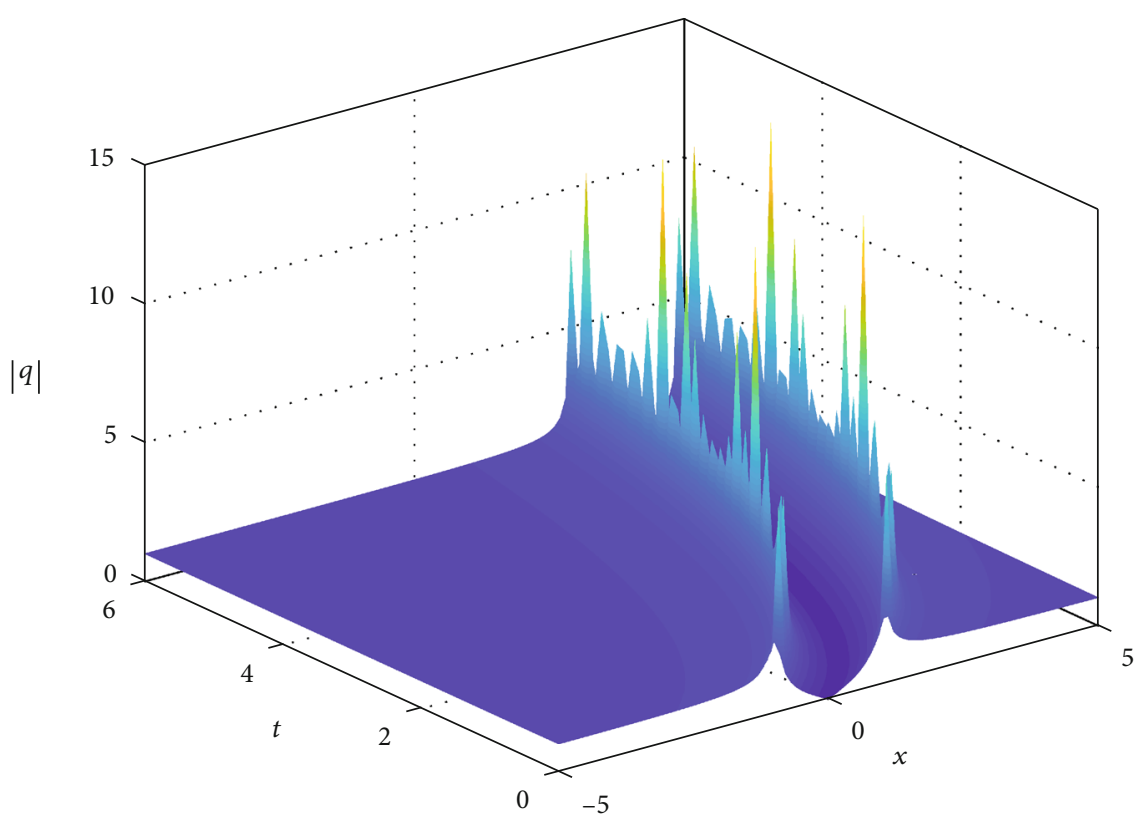

(c)

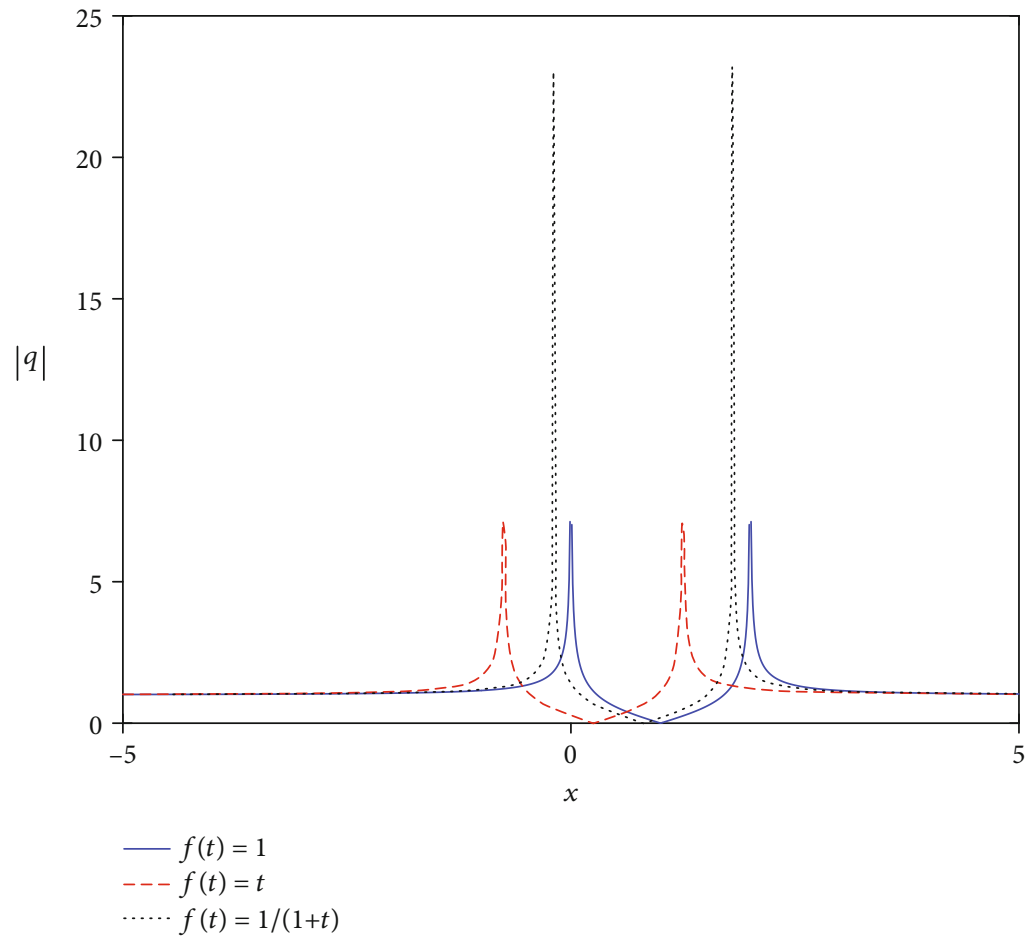

(d)

Figure 6: (a-c) The 3D wave shapes of $|q|:($ a) $f(t)=1$, (b) $f(t)=t$, and (c) $f(t)=1 /(1+t)$. (d) The wave propagates along the $x$-axis. 
$G(\varphi)=0$ has a single root $\phi_{1}=-a_{2} / 3 a_{0}-\left(\sqrt[3]{Y_{1}}+\sqrt[3]{Y_{2}}\right) / 3$, where $Y_{1,2}=A a_{2} / a_{0}+3\left(-B \pm \sqrt{B^{2}-4 A C}\right) / 2$. One has $\phi^{\prime}= \pm 2 \sqrt{a_{0}\left(\phi-\phi_{1}\right)\left(\phi^{2}+\alpha \phi+\beta\right)}$, where two parameters $\alpha$ and $\beta$ are given by $\alpha=2 a_{2} / 3 a_{0}-\left(\sqrt[3]{Y_{1}}+\sqrt[3]{Y_{2}}\right) / 3$ and
$\beta=\left(\sqrt[3]{Y_{1}}+\sqrt[3]{Y_{2}}-2 a_{2} / a_{0}\right)^{2} / 36+\left(\sqrt[3]{Y_{1}}-\sqrt[3]{Y_{2}}\right)^{2} / 12$, respectively. The periodic solutions including an elliptic function are explicitly expressed as

$$
q=\left\{\begin{array}{l}
{\left[\frac{1+\operatorname{cn}\left(2 \varepsilon \sqrt{a_{0}}\left(\phi_{1}^{2}+\alpha \phi_{1}+\beta\right)^{1 / 4} \xi, m_{3}\right)}{\left(\phi_{1}+\sqrt{\phi_{1}^{2}+\alpha \phi_{1}+\beta}\right)+\left(\phi_{1}-\sqrt{\phi_{1}^{2}+\alpha \phi_{1}+\beta}\right) \operatorname{cn}\left(2 \varepsilon \sqrt{a_{0}}\left(\phi_{1}^{2}+\alpha \phi_{1}+\beta\right)^{1 / 4} \xi, m_{3}\right)}\right]^{1 / 2} \cdot e^{i \eta}, \quad a_{0}>0,} \\
{\left[\frac{1+\operatorname{cn}\left(2 \varepsilon \sqrt{-a_{0}}\left(\phi_{1}^{2}+\alpha \phi_{1}+\beta\right)^{1 / 4} \xi, m_{4}\right)}{\left(\phi_{1}-\sqrt{\phi_{1}^{2}+\alpha \phi_{1}+\beta}\right)+\left(\phi_{1}+\sqrt{\phi_{1}^{2}+\alpha \phi_{1}+\beta}\right) \operatorname{cn}\left(2 \varepsilon \sqrt{-a_{0}}\left(\phi_{1}^{2}+\alpha \phi_{1}+\beta\right)^{1 / 4} \xi, m_{4}\right)}\right]^{1 / 2} \cdot e^{i \eta} \quad a_{0}<0,}
\end{array}\right.
$$

where

$$
\begin{aligned}
& m_{3}=\frac{1}{2}\left(2-\frac{2 \varphi_{1}+\alpha}{\sqrt{\varphi_{1}^{2}+\alpha \varphi_{1}+\beta}}\right)^{1 / 2}, \\
& m_{4}=\frac{1}{2}\left(2+\frac{2 \varphi_{1}+\alpha}{\sqrt{\varphi_{1}^{2}+\alpha \varphi_{1}+\beta}}\right)^{1 / 2} .
\end{aligned}
$$

In similar way, the corresponding shapes of the wave amplitude are plotted in Figure 1 (the parameters $k=s=1$, $a_{0}=1, a_{2}=-1, a_{4}=-1$, and $a_{6}=-2$ are chosen here). Notice that these sharp shapes are slightly different from those in Figure 2.

\section{Case 3. $\Delta=0$}

$A \neq 0$

$G(\varphi)=0$ has two roots, $\varphi_{1}=-B / 2 A$ and $\varphi_{2}=-a_{2} / a_{0}+B$ /A. It is known that Equation (12) can be transformed into $\phi^{\prime}= \pm 2 \sqrt{a_{0}\left(\phi-\phi_{1}\right)^{2}\left(\phi-\phi_{2}\right)}$, from which we are able to obtain the exact solutions to Equation (1).

$q=\frac{1}{\left[\phi_{2} \sec ^{2}\left(\sqrt{a_{0}\left(\phi_{2}-\phi_{1}\right)} \xi\right)-\phi_{1} \tan ^{2}\left(\sqrt{a_{0}\left(\phi_{2}-\phi_{1}\right)} \xi\right)\right]^{1 / 2}} \cdot e^{i \eta}, a_{0} \phi_{1}<a_{0} \phi_{2}$,

$q=\frac{1}{\left[\phi_{2} \sec h^{2}\left(\sqrt{a_{0}\left(\phi_{1}-\phi_{2}\right)} \xi\right)+\phi_{1} \tan h^{2}\left(\sqrt{a_{0}\left(\phi_{1}-\phi_{2}\right)} \xi\right)\right]^{1 / 2}} \cdot e^{i \eta}, a_{0} \phi_{1}>a_{0} \phi_{2}$.

For the case $a_{0} \varphi_{1}<a_{0} \varphi_{2}$, the shapes of the periodic wave amplitude are shown in Figure $3\left(k=s=1, a_{0}=1, a_{2}=-4\right.$, $a_{4}=5$, and $\left.a_{6}=-2\right)$, which is very similar to those in Figure 1. For the case $a_{0} \varphi_{1}>a_{0} \varphi_{2}\left(a_{0}>0\right)$, the shapes of the wave amplitude are displayed in Figure $4\left(k=s=1, a_{0}=1\right.$, $a_{2}=-5, a_{4}=8$, and $a_{6}=-4$ ), which corresponds to a bright soliton wave. However, the case $a_{0}<0$ generates a dark soli- ton wave as shown in Figure $5\left(k=s=1, a_{0}=-1, a_{2}=4, a_{4}\right.$ $=-5$, and $a_{6}=2$ ). These new and interesting phenomena do not appear in other cases due to the different signs of the parameter $a_{0}$.

$$
\begin{aligned}
& A=0 \text { and } B=0, \text { i.e., } a_{4}=a_{2}^{2} / 3 a_{0}, a_{6}=a_{2}^{3} / 27 a_{0}^{2} \\
& G(\varphi)=0 \text { has three equal roots, } \varphi_{1}=-a_{2} / 3 a_{0} . \text { The relation }
\end{aligned}
$$
$\varphi^{\prime}= \pm 2 \sqrt{a_{0}\left(\varphi-\varphi_{1}\right)^{3}}$ enables us to obtain a rational function solution

$$
q=\left(\frac{3 a_{0} \xi^{2}}{3-a_{2} \xi^{2}}\right)^{1 / 2} \cdot e^{i \eta}
$$

which represents a singular solitary wave. The corresponding shapes of the wave amplitude are illustrated in Figure 6 $\left(k=s=1, a_{0}=1, a_{2}=3, a_{4}=3\right.$, and $\left.a_{6}=1\right)$.

Before ending this section, we give a useful remark on these graphics. Comparing Figures 2(a)-2(c), it is found that the amplitude is the same. This implies that the variable coefficient $f(t)$ has no effect on the amplitude. We have the same conclusion for Figures 1 and 3-5, but the amplitude in Figure 6 is changed.

\section{Concluding Remarks}

In this paper, we investigated a kind of nonlinear Schrödinger equation with high-order time-dependent coefficients, which describes the propagation of ultrashort optical pulses in nonlinear optical fibers. The trial function method has been used to find general solutions such as the periodic solutions (14) and (16) including the Jacobi elliptic function, the periodic wave solutions (18) involving the trigonometric function, solitary wave solutions (19), and rational function solutions (20). To describe the properties of the solutions, three different functions, $f(t)=1, f(t)=t$, and $f(t)=1 /(1$ $+t$ ), are chosen to show the shapes of the wave amplitudes. We summarize the main results as follows: 
(1) For $f(t)=1$, Figures 1(a), 2(a), and 3(a) show the shapes of the wave amplitude of the periodic wave solutions (14)-(18), respectively. Figures 4(a) and 5(a) display the case of the solitary wave solutions (19); Figure 4(a) shows the case of a bright soliton $\left(a_{0}>0\right)$, but Figure 5(a) illustrates the case of a dark soliton $\left(a_{0}<0\right)$. Figure 6(a) shows the wave shapes of the rational function solutions (20). By observing Figures 1(a), 2(a), 3(a), 4(a), 5(a), and 6(a), it is found that the velocity of the pulse remains constant during propagation because $f(t)$ is a real constant

(2) For the case $f(t)=t$, the shapes of the wave amplitude of the periodic wave solutions (14)-(18) are shown in Figures 1(b), 2(b), and 3(b), respectively. The case of the solitary wave solutions (19) is plotted in Figures 4(b) and 5(b). For example, Figure 4(b) describes a bright soliton wave $\left(a_{0}>0\right)$, while Figure 5(b) corresponds to a dark soliton wave $\left(a_{0}<0\right)$. The shape of the wave amplitude of the rational function solutions (20) is illustrated in Figure 6(b). It can be found that the pulse propagation velocity has a parabolic feature by observing Figures 1(b), 2(b), 3(b), 4(b), 5(b), and 6(b).

(3) For $f(t)=1 /(1+t)$, Figures 1(c), 2(c), and 3(c) describe the periodic wave solutions (14)-(18), respectively. Figures $4(\mathrm{c})$ and 5(c) show the case of the solitary wave solutions (19). For instance, Figure 4(c) represents a bright soliton wave $\left(a_{0}>0\right)$, but Figure 5(c) corresponds to a dark soliton wave $\left(a_{0}<0\right)$. Figure 6(c) shows the shapes of the wave amplitude of the rational function solutions (20). It is seen from Figures 1(c), 2(c), 3(c), 4(c), 5(c), and 6(c) that the pulse propagation has the feature of a logarithmic function.

Before ending this work, we will make three useful remarks. First, it should be mentioned that the specific expressions of various exact solutions of the NLSE with varying coefficients also reflect the diversity of the solitary wave solutions. The existence of solitary wave solutions implies a perfect balance between the nonlinear effect and the dispersion effect, which usually requires peculiar conditions. Without doubt, this work will help us understand the physical phenomena described by Equation (1). Second, it is believed that the results such as optical solitons presented here will make a major impact in the area of nonlinear optics. The model which was used in this work for mathematical analysis gave the characteristics of stable solitary waves in the system. This confirms that the optical fiber described by the system can be transmitted stably for a long time. Third, the trial function method is also an effective and practical method for solving other kinds of nonlinear equations with varying coefficients.

\section{Data Availability}

The data used to support the findings of this study are available from the corresponding author upon request.

\section{Conflicts of Interest}

The authors declare that there is no conflict of interest regarding the publication of this paper.

\section{Funding}

This work was partially supported by 20210414-SIP-IPN.

\section{References}

[1] W. Bao, "The nonlinear Schrödinger equation and applications in Bose-Einstein condensation and plasma physics," Dynamics in Models of Coarsening, Coagulation, Condensation and Quantization, vol. 9, pp. 141-240, 2007.

[2] T. B. Benjamin and J. E. Feir, "The disintegration of wave trains on deep water," Journal of Fluid Mechanics, vol. 27, no. 3, pp. 417-430, 1967.

[3] G. B. Whitham, "Non-linear dispersive waves," Proceedings of the Royal Society of London, Series A, Mathematical and Physical Sciences, vol. 283, no. 1393, pp. 238-261, 1965.

[4] J. L. Rosales and J. L. Sánchez-Gómez, "Non-linear Schrodinger equation coming from the action of the particle's gravitational field on the quantum potential," Physics Letters A, vol. 166, no. 2, pp. 111-115, 1992.

[5] C. Coste, "Nonlinear Schrödinger equation and superfluid hydrodynamics," The European Physical Journal BCondensed Matter and Complex Systems, vol. 1, no. 2, pp. 245-253, 1998.

[6] M. Wang, Y. Zhou, and Z. Li, "Application of a homogeneous balance method to exact solutions of nonlinear equations in mathematical physics," Physics Letters A, vol. 216, no. 1-5, pp. 67-75, 1996.

[7] M. Yaghobi Moghaddam, A. Asgari, and H. Yazdani, "Exact travelling wave solutions for the generalized nonlinear Schrodinger (GNLS) equation with a source by Extended tanh-coth, sine-cosine and Exp- Function methods," Applied Mathematics and Computation, vol. 210, no. 2, pp. 422-435, 2009.

[8] W. X. Ma, T. Huang, and Y. Zhang, "A multiple exp-function method for nonlinear differential equations and its application," Physica Scripta, vol. 82, no. 6, article 065003, 2010.

[9] N. Taghizadeh, M. Mirzazadeh, and F. Farahrooz, "Exact solutions of the nonlinear Schrodinger equation by the first integral method," Journal of Mathematical Analysis and Applications, vol. 374, no. 2, pp. 549-553, 2011.

[10] M. Arshad, A. R. Seadawy, and D. Lu, "Elliptic function and solitary wave solutions of the higher-order nonlinear Schrödinger dynamical equation with fourth-order dispersion and cubic-quintic nonlinearity and its stability," The European Physical Journal Plus, vol. 132, no. 8, p. 371, 2017.

[11] M. Wang, X. Li, and J. Zhang, "Sub-ODE method and solitary wave solutions for higher order nonlinear Schrödinger equation," Physics Letters A, vol. 363, no. 1-2, pp. 96-101, 2007.

[12] M. Wang, X. Li, and J. Zhang, "The $\left(G^{\prime} / G\right)$-expansion method and travelling wave solutions of nonlinear evolution equations in mathematical physics," Physics Letters A, vol. 372, no. 4, pp. 417-423, 2008.

[13] A. J. M. Jawad, M. D. Petković, and A. Biswas, "Modified simple equation method for nonlinear evolution equations," Applied Mathematics and Computation, vol. 217, no. 2, pp. $869-877,2010$. 
[14] G. Xu, "Extended auxiliary equation method and its applications to three generalized NLS equations," Abstract and Applied Analysis, vol. 2014, Article ID 541370, 7 pages, 2014.

[15] K. Khan and M. A. Akbar, "Application of $\exp (-\Phi(\xi))$-expansion method to find the exact solutions of modified BenjaminBona-Mahony equation," World Applied Sciences Journal, vol. 24, no. 10, pp. 1373-1377, 2013.

[16] C. Rui and Z. Jian, “Trial function method and exact solutions to the generalized nonlinear Schrödinger equation with timedependent coefficient," Chinese Physics B, vol. 22, no. 10, article 100507, 2013.

[17] H. Triki and A. M. Wazwaz, "Soliton solutions of the cubicquintic nonlinear Schrödinger equation with variable coefficients," Romanian Journal of Physics, vol. 61, no. 3-4, p. 360, 2016.

[18] E. M. E. Zayed and M. A. M. Abdelaziz, "Exact solutions for the nonlinear Schrodinger equation with variable coefficients using the generalized extended tanh-function, the sine-cosine and the exp-function methods," Applied Mathematics and Computation, vol. 218, no. 5, pp. 2259-2268, 2011.

[19] Q. Guo and J. Liu, "New exact solutions to the nonlinear Schrodinger equation with variable coefficients," Results in Physics, vol. 16, p. 102857, 2020.

[20] C. Liu, Y. Y. Li, M. Gao, Z. Wang, Z. Dai, and C. Wang, "Rogue wave solutions of the nonlinear Schrödinger equation with variable coefficients," Pramana, vol. 85, no. 6, pp. 1063-1072, 2015.

[21] Y. Liu, "Exact solutions to nonlinear Schrodinger equation with variable coefficients," Applied Mathematics and Computation, vol. 217, no. 12, pp. 5866-5869, 2011.

[22] P. D. Green and A. Biswas, "Bright and dark optical solitons with time-dependent coefficients in a non- Kerr law media," Communications in Nonlinear Science and Numerical Simulation, vol. 15, no. 12, pp. 3865-3873, 2010.

[23] S.-H. Dong, Wave Equations in Higher Dimensions, Springer, Netherlands, 2011.

[24] R. Castro López, G.-H. Sun, O. Camacho-Nieto, C. Yáñz-Márquez, and S.-H. Dong, "Analytical traveling-wave solutions to a generalized Gross-Pitaevskii equation with some new time and space varying nonlinearity coefficients and external fields," Physics Letters A, vol. 381, no. 35, pp. 2978-2985, 2017.

[25] F. Dalfovo, S. Giorgini, L. P. Pitaevskii, and S. Stringari, "Theory of Bose-Einstein condensation in trapped gases," Reviews of Modern Physics, vol. 71, no. 3, pp. 463-512, 1999.

[26] L. P. Pitaevskii and S. Stringari, Bose-Einstein Condensation, Oxford University Press, Oxford, UK, 2003.

[27] P. G. Kevrekidis, D. J. Frantzeskakis, and R. Carretero-Gonzalez, Emergent Nonlinear Phenomena in Bose-Einstein Condensates: Theory and Experiment, Springer, New York, NY, USA, 2008.

[28] Y. V. Kartashov, B. A. Malomed, and L. Torner, "Solitons in nonlinear lattices," Reviews of Modern Physics, vol. 83, no. 1, pp. 247-305, 2011.

[29] F. K. Abdullaev, A. M. Kamchatnov, V. V. Konotop, and V. A. Brazhnyi, "Adiabatic dynamics of periodic waves in BoseEinstein condensates with time dependent atomic scattering length," Physical Review Letters, vol. 90, no. 23, p. 230402, 2003. 